seizures and epilepsy following a complex FS (see Pediatr Neurol Briefs August 2011;25(8):59). The risk of epilepsy following a simple FS is about 2\% (Nelson KB, Ellenberg JH. N Engl J Med 1976;295:1029-1033) whereas the risk following a complex FS in a child with developmental delay is 9.2\% (Hesdorffer DC et al. Ann Neurol 2011;70(1):93-100). In children with normal development and simple FS, the risk is only $1.1 \%$ but significantly greater than for healthy children with no FS $(0.5 \% ; p=0.027)$. In a prospective study of 428 children with a first FS followed for 2 years or more, unprovoked seizures occurred in $26(6 \%)$. Risk factors for unprovoked seizures included neurodevelopmental abnormalities, complex FS, a family history of epilepsy, recurrent FS, and brief duration of fever before initial FS. (Berg AT, Shinnar S. Neurology 1996;47(2):562-568).

A review of the literature from 1947 to 1964 uncovered 36 publications in which the EEG findings were reported in relation to FS and 23 included number and percent with paroxysmal discharges (mean 25\%). (Millichap JG. Febrile Convulsions. New York, Macmillan, 1968). In our prospective 2-year follow-up study of 76 FS patients with EEGs, paroxysmal discharges were recorded in $18(24 \%)$; they were more frequent in patients who developed non-FS (61\%) than in those with FS alone (12\%). The discharges were generalized in 11 patients and focal in 7 (Millichap JG et al. Neurology 1960;10:643-653). Age was a significant factor in relation to the incidence of abnormal EEGs; patients with abnormal records were 3-10 years (mean 7 years) old and those with normal records were 1-7 years (mean 3 years) old. In her extensive investigations of the EEG and FS, Lennox MA also reported that paroxysmal records occurred mainly in children 5 years or older (Amer J Dis Child 1949;78:868-882). Repeated EEGs at follow-up subsequent to a FS were recommended to determine prognosis and risk of nonfebrile seizures. The present study concludes with a similar recommendation, but more specifically in patients with EEG showing frontal paroxysmal foci.

\title{
NONCONVULSIVE ELECTROGRAPHIC STATUS EPILEPTICUS
}

Researchers at the University of Cincinnati, $\mathrm{OH}$, examined medical records of 75 children, aged 3 months to 21 years (mean age 7.8 years), for prevalence of nonconvulsive status epilepticus (NCSE) by searching a clinical EEG database $(n=18)$ or consecutive inpatient EEG referrals for NCSE over an 8-month period $(n=57)$. NCSE was identified in 26 patients (35\%) and in 8 of $57(14 \%)$ patients referred for possible NCSE ( $>50 \%$ from outside the ICU). An acute etiology for encephalopathy was determined in 31 of $75(41 \%)$ patients; it was an extra CNS infection and fever in 12, CNS infection in 8 , and hypoxia in 12. Less than half the patients with NCSE were critically ill; 4 NCSE patients $(15 \%)$ died, and $8(31 \%)$ had significant neurologic morbidity on discharge. Compared to patients identified with NCSE, of the 49 patients without NCSE, $4(8 \%)$ died and $2(4 \%)$ had neurologic morbidity. The majority of patients (15 of 26, 58\%) identified with NCSE were in the neurology service. A clinical seizure was witnessed in $24(92 \%)$ patients with NCSE. Of 57 patients with acute neuroimaging within 24 hours of EEG, 20 showed abnormalities including multifocal cortical edema and acute hydrocephalus. Clinical seizures and acute neuroimaging abnormality were associated with an $82 \%$ probability of NCSE. NCSE was accompanied by electrographic or electroclinical seizures within the first hour of monitoring; the median duration of 
monitoring was 11.5 hours. Continuous EEG monitoring is recommended in children with acute encephalopathy. (Greiner HM, Holland K, Leach JL, Horn PS, Hershey AD, Rose DF. Nonconvulsive status epilepticus: the encephalopathic pediatric patient. Pediatrics March 2012;129(3):e748-55). (Respond: Hansel M Greiner MD, Cincinnati Children's Hospital Medical Center, 3333 Burnet Ave, MLC 2015, Cincinnati, OH 45229. E-mail: hansel.greiner@cchmc.org).

COMMENT. Risk factors for NCSE in children include a prior clinical seizure and acute cortical imaging abnormality. When both of these variables were present, the probability of NCSE was high (82\%). If NCSE is suspected, continuous EEG monitoring is important not only in high-risk neonates in the ICU but also in children and adolescents with disturbed consciousness and symptoms of encephalopathy. The Cincinnati investigators defined NCSE as continuous 30-minute electrographic seizure activity with non-convulsive clinical symptoms or repeated briefer electrographic seizures comprising at least 30 minutes of a 1-hour period (Greiner HM et al. 2012).

A NCSE etiological classification included metabolic disorders, coma, acute cerebral lesions, and preexisting epilepsy (Maganti $R$ et al. Epilepsy Behav 2008;12(4):572-586). NCSE constitutes about 25-50\% of all cases of status epilepticus. In comatose patients, NCSE diagnosis is often difficult and potentially fatal if untreated. The EEG may show a variety of rhythmic or periodic patterns, some of unclear significance. Of 19 pediatric patients with NCSE identified from the database of the Columbia University Epilepsy Center, 6 had periodic lateralized epileptiform discharges (PLEDS), and 1 had generalized PEDS. Periodic discharges were associated with worse outcome. (Tay SK et al. Epilepsia 2006;47(9):1504-1509). The most frequent etiology of NCSE in this study was acute hypoxic-ischemic encephalopathy (26\%); other causes included metabolic (21\%), infection (16\%), AED change (16\%), refractory epilepsy $(11 \%)$, and intracranial hemorrhage (11\%). Prompt recognition of NCSE by continuous EEG monitoring should lead to early treatment and improved prognosis.

\section{HIGH-FREQUENCY EEG OSCILLATIONS: NEW BIOMARKER IN EPILEPSY}

Researchers at University Medical Center Utrecht, The Netherlands and other centers review the pathophysiology, clinical relevance, identification, and interpretation of high-frequency oscillations (HFOs, $>80 \mathrm{~Hz}$ ) in the epileptic EEG. HFOs are further classified in ripples $(80-250 \mathrm{~Hz})$, fast ripples $(250-600 \mathrm{~Hz})$, and very-HFOs $(>1,000 \mathrm{~Hz})$. HFOs are observed between seizures, at seizure onset, and during seizures. Interictal HFOs occur during slow-wave sleep. The intracranial EEG is sampled at $=>>2,000 \mathrm{~Hz}$. In patients with focal epilepsy who may benefit from surgery, removal of brain tissue generating HFOs results in better outcome than removal of the seizure onset zone. Interictal HFOs are reliable markers of the seizure onset zone, and better than epileptic spikes. HFOs occur in different types of epilepsy, mesiotemporal with hippocampal sclerosis and also in extratemporal epilepsies with tumors, focal cortical dysplasia, and nodular heterotopia. HFOs are sometimes recorded in nonlesional epilepsies. Evaluation of seizures with HFO recordings $>80 \mathrm{~Hz}$ may improve the pre-surgical workup and outcome and reduce the necessity for invasive monitoring. (Zijlmans M, Jiruska P, 